\title{
TRABAJOS GANADORES PRESENTADOS EN EL XXII CONGRESO FLASOG REALIZADO DEL 5 AL 9 DE NOVIEMBRE DE 2017 EN CANCÚN, MÉXICO
}

\section{Prize-winning work presented at the 22nd FLASOG Congress held between the 5th and 9 th of November 2017 in Cancún, México}

\section{COMPARACIÓN DE LA EFECTIVIDAD Y SEGURIDAD DE LA FIJACIÓN SACRO- ESPINOSA UNILATERAL VERSUS BILATERAL, EN EL TRATAMIENTO DEL PROLAPSO DE CÚPULA VAGINAL. ENSAYO CLÍNICO CONTROLADO Y ALEATORIZADO}

\section{Franklin José Espitia De La Hoz, Servicio de Ginecología y Medicina Materno Fetal, Clínica La Sagrada Familia, Armenia (Colombia). espitiafranklin@hotmail.com}

\section{RESUMEN}

Introducción: el prolapso de los órganos pélvicos es común y se define como la herniación de los órganos pélvicos a través de la vagina. La incidencia del prolapso de cúpula vaginal, luego de la histerectomía, no es bien conocida, y aunque el tratamiento es individualizado, el propósito es la creación de un nuevo punto de suspensión, a fin de cubrir el diafragma pélvico. En esto, la técnica de fijación sacro-espinosa ha demostrado ser ventajosa.

Objetivo: comparar la efectividad y seguridad entre las pacientes sometidas a fijación unilateral de los ligamentos sacro-espinosos contra la fijación bilateral, para el tratamiento del prolapso apical poshiste- rectomía. Se realizó un ensayo clínico, aleatorizado y controlado, no enmascarado. Se asignaron dos grupos aleatorizados: grupo A (50 mujeres operadas con técnica de fijación unilateral al ligamento sacro espinoso,) y grupo B (46 mujeres operadas con técnica de fijación bilateral al ligamento sacro-espinoso). El estudio fue realizado entre enero de 2009 a diciembre de 2015, en la consulta externa de la Clínica La Sagrada Familia de Armenia, Quindío (Colombia), Suramérica. Participaron 96 mujeres con prolapso de cúpula vaginal. Las principales medidas de resultados fueron: longitud vaginal total (tvl), el punto $\mathrm{C}$, tiempo quirúrgico, cantidad de sangrado, tasa de éxito, complicaciones, satisfacción, cura subjetiva y satisfacción sexual. Resultados: la edad promedio de las pacientes fue de $69 \pm 4,83$ años. El intervalo de tiempo medio de seguimiento posterior a la cirugía fue de 54 meses (rango 15-72). La fijación unilateral demanda un tiempo más corto de operación (42 \pm 18 minutos frente a $81 \pm 24$ minutos, $\mathrm{p}=0,008)$, con una media de sangrado menor $(117 \pm 72 \mathrm{~mL}$ frente a $261 \pm 93 \mathrm{~mL}, \mathrm{p}=0,009)$. A los 54 meses no se encontraron diferencias en las tasas de éxito (fijación unilateral $84 \%$ frente a 89,13\% de fijación bilateral). La media del punto $\mathrm{C}$ y la longitud vaginal, a 72 meses, fue de $-5,75 \pm 1,3$ y 7,0 $\pm 1,4$, respectivamente, para la fijación unilateral, y de $-6,86 \pm 0,73$ y 7,5 $\pm 1,5$, respectivamente, para la fijación bilateral. La recurrencia en los compartimentos anterior o posterior 
fue del $12 \%$ frente a 10,86\%, en la fijación unilateral, y $8 \%$ frente a $6,52 \%$, respectivamente, en la fijación bilateral. Conclusiones: la fijación a los ligamentos sacro-espinosos es una opción efectiva y segura para la corrección del prolapso de cúpula.

Palabras clave: atrofia, cirugía, clasificación, prolapso, sacro, tratamiento.

ESTADO DE LA VITAMINA D EN GESTANTES COLOMBIANAS CON PREECLAMPSIA Y NORMOTENSAS: ESTUDIO PREVITD DE CASOS Y CONTROLES A GRAN ESCALA

\section{Norma Serrano, Elizabeth Guío, Dora Cristina Quintero-Lesmes, Silvia Milena Becerra, Carlos Prada, María Luna, Juan Pablo Casas, Fundación Cardiovascular de Colombia. Bucaramanga, Colombia. normaserrano@fcv.org}

Introducción: los datos epidemiológicos han demostrado que la deficiencia de vitamina D (VD) durante el embarazo provoca un riesgo mayor para desarrollar preeclampsia (PE). No obstante, esta evidencia es conflictiva y algunos de los estudios están limitados por tamaño de muestra, ausencia de ajuste por factores de confusión y por no contemplar todos los grupos poblacionales.

Objetivo: comprobar si niveles bajos de VD definida como $25(\mathrm{OH}) \mathrm{D}<30 \mathrm{ng} / \mathrm{mL}$ se asocian con un riesgo mayor de desarrollar PE en gestantes colombianas. Metodología: se analizaron en total 1.817 pacientes incluidas en el estudio GenPE (www.genpe.org), 912 con diagnóstico de PE (casos) y 905 gestantes normotensas (controles). Los sueros maternos fueron cuantificados para el total de 25-hidroxivitamina D $(25(\mathrm{OH}) \mathrm{D})[25(\mathrm{OH}) \mathrm{D} 2+25(\mathrm{OH}) \mathrm{D}$ 3] utilizando cromatografía líquida en tándem acoplada a espectrometría de masas.
Las diferencias entre los casos y los controles se evaluaron mediante la prueba U de Mann-Whitney. Se utilizó regresión logística múltiple para evaluar la asociación entre los estados de VD (deficiencia, insuficiencia, suficiencia, bajo nivel) y PE. Para el análisis del subfenotipo se estimó la asociación del biomarcador con la presencia de PE de inicio temprano, tardío y síndrome de HELLP. Para la realización de los cálculos se utilizó el programa estadístico STATA.

Resultados: las concentraciones de VD fueron menores en los casos con respecto a los controles. En las pacientes con PE la media de $25(\mathrm{OH}) \mathrm{D}$ fue de 29,99 ng/mL (IC $95 \%$ : 29,40-30,58). El 14,4\% tenía niveles de $25(\mathrm{OH}) \mathrm{D}$ de $20 \mathrm{ng} / \mathrm{mL}$ o menos, el 37,2\% niveles entre 21-29 ng/mL, el 48,3\% niveles de $30 \mathrm{ng} /$ $\mathrm{mL}$ o más, y el 51,6\% bajo nivel de vitamina $\mathrm{D}(<30$ $\mathrm{ng} / \mathrm{mL})$. En el grupo control, la media de $25(\mathrm{OH}) \mathrm{D}$ materna fue de 33,7 ng/mL (IC 95 \%: 33,20-34,30). El 5,32\% tenía niveles de $25(\mathrm{OH}) \mathrm{D}$ de $20 \mathrm{ng} / \mathrm{mL}$ o menos, el 28,3\% niveles entre 21-29 ng/mL, el 66,3\% niveles de $30 \mathrm{ng} / \mathrm{mL}$ o más, y el 33,6\% bajo nivel de vitamina $\mathrm{D}(<30 \mathrm{ng} / \mathrm{mL})$.

La media de $25(\mathrm{OH})$ D para las paciente con PE tardía fue de 30,4 ng/mL (IC $95 \%: 29,7-31,0$ ), mientras que para PE temprana fue $28,9 \mathrm{ng} / \mathrm{mL}$ (IC $95 \%$ : 27,2-30,6) y para síndrome de HELLP fue de 26,4 ng/mL (IC $95 \%: 23,6-29,3$ ).

$\mathrm{Al}$ analizar por subfenotipos vs. controles, y después de ajustar por variables de confusión, niveles bajos de VD $(<30 \mathrm{ng} / \mathrm{mL})$ se asociaron con un incremento en la probabilidad de desarrollar PE temprana (OR = 2,29; IC $95 \%$ : 1,49-3,52), PE tardía (OR = 2,06; IC $95 \%$ : 1,69-2,52) y síndrome de HELLP (OR $=3,36$; IC $95 \%:$ 1,84-6,13).

Discusión: Colombia se encuentra en la latitud y la longitud de $\mathrm{N} 04^{\circ} 00, \mathrm{O} 72^{\circ} 0$ '0", cubre tanto el hemisferio norte como sur, sin variación estacional, y por lo tanto con mayor incidencia de rayos UVB de longitud de onda apropiada para la producción epidérmica de VD. No obstante, nuestro estudio demuestra niveles bajos de VD en un porcentaje alto de gestantes colombianas. 
El nivel bajo de VD, definido como $25(\mathrm{OH}) \mathrm{D}<30$ $\mathrm{ng} / \mathrm{mL}$, es un factor de riesgo independiente para el desarrollo de PE en la población estudiada. No obstante, no se puede inferir una relación causal entre la deficiencia de vitamina D y PE debido al diseño observacional de este estudio, pero los resultados son lo suficientemente fuertes para proponer la conducción de un ensayo clínico controlado, bien definido, que logre identificar la ventana de tiempo crítico y la dosis más eficaz de VD para prevenir el desarrollo de PE y, por tanto, establecer un potencial terapéutico de la VD en la gestación, si lo hay.
Conclusión: nuestros resultados, en una muestra robusta de pacientes, apoyan la plausibilidad biológica que tiene la VD durante la gestación. Sin embargo, se necesitan más investigaciones para determinar su papel causal en el desarrollo, la progresión y la severidad de la PE.

Financiación. Esta investigación fue apoyada por Colciencias, según Grant de investigación 492 de 2012, y FARMA de Colombia, patrocinio no condicionado, FMN8949. 